\title{
THE PSEUDO-ORBIT TRACING PROPERTY AND EXPANSIVENESS ON THE CANTOR SET
}

\author{
TAKASHI SHIMOMURA
}

(Communicated by Kenneth R. Meyer)

\begin{abstract}
The set of all the expansive homeomorphisms with the pseudo-orbit tracing property is dense in the space of all the homeomorphisms of the Cantor set with the topology of uniform convergence. Moreover a topologically transitive (resp. mixing) homeomorphism of the Cantor set is approximated uniformly by topologically transitive (resp. mixing) expansive homeomorphisms with the pseudo-orbit tracing property.
\end{abstract}

\section{INTRODUCTION}

Let $\mathscr{H}$ be the space of all the homeomorphisms of the Cantor set $C$ in $[0,1]$ with the topology of uniform convergence. It was shown by M. Sears [3] that the set $\mathscr{E}$ of all the expansive homeomorphisms of $C$ is dense in $\mathscr{H}$. And M. Dateyama [2] showed that the set $\mathscr{P}$ of all the homeomorphisms of $C$ with the pseudo-orbit tracing property (abbrev. POTP) is dense in $\mathscr{H}$. The purpose of this paper is to show that the set $\mathscr{S}$ of all the homeomorphisms which are topologically conjugate to subshifts of finite type is also dense in $\mathscr{H}$. Since $\mathscr{S}=\mathscr{E} \cap \mathscr{P}$ as being shown later, it is a generalization of the results above.

Given an integer $r \geq 1$, we call $\left[i 3^{-r},(i+1) 3^{-r}\right] \cap C,\left(i=0,1, \ldots, 3^{-r}-1\right)$ a Cantor subinterval of rank $r$ if $\left(i \cdot 3^{-r},(i+1) \cdot 3^{-r}\right) \cap C \neq \varnothing$. Order the subintervals of rank $r$ by the usual ordering of their left-hand endpoints and denote the $k$ th in this order by $I(k, r) \quad\left(k=1,2, \ldots, 2^{r}\right)$. Note that $\operatorname{diam} I(k, r)=3^{-r}$. A Cantor subinterval is homeomorphic to $C$. More generally, a compact metrizable totally disconnected perfect space is homeomorphic to $C$.

Let $n$ be a positive integers and let $S_{n}=\{1,2, \ldots, n\}$ with the discrete topology. We put

$$
\Sigma_{n}=\left\{x ; x=\left(x_{i}\right)_{i \in \mathbf{Z}}, x_{i} \in S_{n}(i \in \mathbf{Z})\right\}
$$

Received by the editors March 6, 1988 and, in revised form, May 30, 1988.

1980 Mathematics Subject Classification (1985 Revision). Primary 54H20, 34C35.

Key words and phrases. Cantor set, homeomorphism, the pseudo-orbit tracing property, expansive, subshift of finite type, subshift. 
with the product topology. Then $\Sigma_{n}$ is a compact metrizable totally disconnected perfect space. The shift map $\sigma_{n}: \Sigma_{n} \rightarrow \Sigma_{n}$ is defined by $\left(\sigma_{n}(x)\right)_{i}=x_{i+1}$ $(i \in \mathbf{Z})$, where $x=\left(x_{i}\right)_{i \in \mathbf{Z}} \in \Sigma_{n}$. Then $\sigma_{n}$ is a homeomorphism and the pair $\left(\Sigma_{n}, \sigma_{n}\right)$ is called a full shift of $n$ symbols. Let $\Lambda \subset \Sigma_{n}$ be a closed $\sigma_{n}$-invariant set, i.e., $\sigma_{n}(\Lambda)=\Lambda$. Then the restriction $\left(\Lambda,\left.\sigma_{n}\right|_{\Lambda}\right)$ of $\sigma_{n}$ on $\Lambda$ is called a subshift. Let $A$ be an $n \times n$ matrix of 0 's and 1's. We put

$$
\Sigma_{A}=\left\{x \in \Sigma_{n} ; x=\left(x_{i}\right)_{i \in \mathbf{Z}}, A_{x_{i}, x_{i+1}}=1, i \in \mathbf{Z}\right\}
$$

and $\sigma_{A}=\left.\sigma_{n}\right|_{\Sigma_{A}}$. Then $\left(\Sigma_{A}, \sigma_{A}\right)$ is a subshift. A subshift $\left(\Lambda,\left.\sigma_{m}\right|_{\Lambda}\right) \quad(m \geq 1)$ is said to be of finite type if it is topologically conjugate to $\left(\Sigma_{A}, \sigma_{A}\right)$ for some $n \times n$ matrix $A$ of 0 's and 1's $(n \geq 1)$.

A homeomorphism $f \in \mathscr{H}$ is topologically transitive if given nonempty open sets $U$ and $V$ of $C, U \cap f^{n}(V) \neq \varnothing$ for some integer $n$. An $f \in \mathscr{H}$ is topologically mixing if given nonempty open sets $U$ and $V$ of $C$, there is an $L>0$ such that $U \cap f^{l}(V) \neq \varnothing$ for all $l \geq L$. An $f \in \mathscr{H}$ is topologically transitive if and only if it has a dense orbit $o_{f}(x) \quad(x \in C)$.

Theorem. $\mathscr{S}$ is dense in $\mathscr{H}$. Moreover, if $f \in \mathscr{H}$ is topologically transitive (resp. mixing), then $f$ is approximated uniformly by elements of $\mathscr{S}$ which are also topologically transitive (resp. mixing).

\section{Preliminaries}

For a homeomorphism of a compact space, the expansiveness and POTP is independent of the metric used. A homeomorphism of a Cantor set is expansive if and only if it is topologically conjugate to a subshift. And a subshift is of finite type if and only if it has POTP (Theorem 1. of P. Walters [4]). Therefore, we get the following

Proposition 1. $\mathscr{E} \cap \mathscr{P}=\mathscr{S}$.

Definition. An $n \times n$ matrix $A$ of 0 's and 1's is irreducible if, for every $a, b$ $(1 \leq a, b \leq n)$, there is an $l>0$ such that $A_{a, b}^{l}>0$, where $A_{a, b}^{l}$ is the $(a, b)$ component of the matrix $A^{l}=A \times \cdots \times A$ ( $l$-times).

Remark. If $A$ is an irreducible $n \times n$ matrix, then, for every $L>0$ and $1 \leq a, b \leq n$, there is an $l \geq L$ such that $A_{a, b}^{l}>0$.

The following Lemma 2 is well known, so we omit a proof.

Lemma 2. Let $A$ be an irreducible $n \times n$ matrix (of 0 's and 1 's). Then for any nonempty open sets $U$ and $V$ of $\Sigma_{A}$, there is an arbitrarily large $m>0$ such that $\sigma_{A}^{m}(U) \cap V \neq \varnothing$.

Lemma 3. Let $A$ be a nondegenerate $n \times n$ matrix of 0 's and 1 's. Then $\left(\Sigma_{A}, \sigma_{A}\right)$ is topologically mixing if and only if there is an $m>0$ such that $A^{m}>0$ (i.e., $A_{a, b}^{m}>0$ for all $1 \leq a, b \leq n$ ).

Proof. Lemma 1.3 of R. Bowen [1]. 


\section{A PROOF OF THE MAIN RESUlT}

The following is a proof of our main result. Let $f \in \mathscr{H}$ and $\varepsilon>0$. Fix an integer $r>0$ with $3^{-r}<\varepsilon / 2$ such that $|x-y| \leq 3^{-r} \quad(x, y \in C)$ implies $|f(x)-f(y)|<\varepsilon / 2 \quad(x, y \in C)$. Let $n=2^{r}$. Define an $n \times n$ matrix $A=A_{f}$ as follows. For $1 \leq a, b \leq n$,

$$
A_{a, b}= \begin{cases}1 & \text { if } f(I(a, r)) \cap I(b, r) \neq \varnothing, \\ 0 & \text { otherwise. }\end{cases}
$$

Then for each $a(1 \leq a \leq n)$, there exists $b$ and $c(1 \leq b, c \leq n)$ such that $A_{b, a}=A_{a, c}=1$. Thus if we put

$$
(a)_{A}=\left\{x \in \Sigma_{A} ; x_{0}=a\right\} \quad(1 \leq a \leq n),
$$

then each $(a)_{A}(1 \leq a \leq n)$ is not empty.

First suppose that each $(a)_{A}(1 \leq a \leq n)$ is perfect. Then there is a homeomorphism $\varphi$ from $\Sigma_{A}$ onto $C$ such that $\varphi\left(\left(a_{A}\right)\right)=I(a, r) \quad(1 \leq a \leq n)$. Put $g=\varphi \cdot \sigma_{A} \cdot \varphi^{-1}$. We shall show that $|f(x)-g(x)|<\varepsilon \quad(x \in C)$. Let $x \in C$. Suppose that $x \in I(a, r)$ and that $g(x) \in I(b, r)$. Then since

$$
\sigma_{A}\left(\varphi^{-1}(x)\right)=\varphi^{-1}(g(x)) \in \sigma_{A}\left((a)_{A}\right) \cap(b)_{A} \neq \varnothing,
$$

we get $A_{a, b}=1$. Hence there is a $y \in I(a, r)$ such that $f(y) \in I(b, r)$. Thus we get

$$
\begin{aligned}
|f(x)-g(x)| & \leq|f(x)-f(y)|+|f(y)-g(x)| \\
& <\varepsilon / 2+3^{-r} \\
& <\varepsilon .
\end{aligned}
$$

In the general case, we shall use a product $\left(\Sigma_{A} \times \Sigma_{2}, \sigma_{A} \times \sigma_{2}\right)$, which is naturally topologically conjugate to a subshift of finite type. Since each $(a)_{A} \times \Sigma_{2}$ $(1 \leq a \leq n)$ is perfect, we get a homeomorphism $\varphi$ from $\Sigma_{A} \times \Sigma_{2}$ to $C$ such that

$$
\varphi\left((a)_{A} \times \Sigma_{2}\right)=I(a, r) \quad(1 \leq a \leq n) .
$$

Putting $g=\varphi \circ\left(\sigma_{A} \times \sigma_{2}\right) \circ \varphi^{-1}$ we proceed as before to get an inequality; $|f(x)-g(x)|<\varepsilon \quad(x \in C)$. Thus we have proved the first half of the Theorem.

We shall show that $g$ is topologically transitive (resp. mixing) when $f$ is topologically transitive (resp. mixing). Suppose that $f$ is topologically transitive. Then there is a dense orbit $o_{f}(x)(x \in C)$. Since each point of $C$ is not isolated, $o_{f}(x)$ is a set of first category. Thus $C-o_{f}(x)$ is also dense in $C$. Since each point of $C-o_{f}(x)$ is a limit point of $o_{f}(x)$, we get $C=\alpha_{f}(x) \cup \omega_{f}(x)$, where $\alpha_{f}(x)$ (resp. $\omega_{f}(x)$ ) is the $\alpha$ (resp. $\omega$-) limit set of $x$ by $f$. Since both $\alpha_{f}(x)$ and $\omega_{f}(x)$ are closed $f$-invariant sets, $U=C-\alpha_{f}(x)$ and $V=C-\omega_{f}(x)$ are disjoint open $f$-invariant sets. Thus, by topological transitivity, either $U$ or $V$ must be empty. Hence either $\alpha_{f}(x)=C$ or $\omega_{f}(x)=C$ holds. In either case, for any $1 \leq a, b \leq n$, there 
is an $l_{a, b}>0$ such that $f^{l_{a, b}}(I(a, r)) \cap(I(b, r)) \neq \varnothing$. Then it is easy to check that $A_{a, b}^{l_{a, b}}>0(1 \leq a, b \leq n)$, where $A=A_{f}$. Thus $A\left(=A_{f}\right)$ is irreducible. Since $\left(\Sigma_{2}, \sigma_{2}\right)$ is topologically mixing, both $\left(\Sigma_{A}, \sigma_{A}\right)$ and $\left(\Sigma_{A} \times \Sigma_{2}, \sigma_{A} \times \sigma_{2}\right)$ are topologically transitive by Lemma 2 . Hence $g$ is topologically transitive in either case. Next suppose that $f$ is topologically mixing. It is enough to show that $\left(\Sigma_{A}, \sigma_{A}\right)$ is topologically mixing and that $\Sigma_{A}$ is perfect. For any $1 \leq a, b \leq n$, there is an $L_{a, b}>0$ such that $f^{l}(I(a, r)) \cap I(b, r) \neq \varnothing$ for all $l \geq L_{a, b}$. Thus $A_{a, b}^{l}>0$ for all $l \geq L_{a, b} \quad(1 \leq a, b \leq n)$. Hence $\left(\Sigma_{A}, \sigma_{A}\right)$ is topologically mixing, by Lemma 3 . Suppose that $\Sigma_{A}$ has an isolated point $p$. Then, since $\{p\}$ is an open set, there is an $L>0$ such that $p=f^{l}(p)$ for all $l \geq L$. Thus $p$ is a fixed point. This contradicts the fact that $\left(\Sigma_{A}, \sigma_{A}\right)$ is topologically mixing, for $\Sigma_{A}-\{p\}$ is also open and $f$-invariant.

Remark. In the above proof, one could use another perfect subshift say $(\Sigma, \sigma)$ in place of $\left(\Sigma_{2}, \sigma_{2}\right)$. If a property of $(\Sigma, \sigma)$ is not lost by taking a product with any subshift of finite type $\left(\Sigma_{A}, \sigma_{A}\right)$, then elements of $\mathscr{H}$ will be approximated uniformly by subshifts with this property.

After I finished writing this paper, I accepted T. Kimura [4], where the density of $\mathscr{E} \cap \mathscr{P}$ in $\mathscr{H}$ is proved independently.

\section{ACKNOWLEDGMENTS}

The author would like to express his thanks to Professor N. Aoki, Professor M. Dateyama and Professor K. Shiraiwa for valuable conversations and suggestions in forming this paper, and the referee for kind advices.

\section{REFERENCES}

1. R. Bowen, Equilibrium states and the ergodic theory of Anosov diffeomorphisms, Lecture Notes in Math., vol. 470, Springer-Verlag, Berlin, Heidelberg and New York, 1975.

2. M. Dateyama, Homeomorphisms with the pseudo-orbit tracing property of the Cantor set, Tokyo J. Math. 6 (1983), 287-290.

3. M. Sears, Expansive self-homeomorphisms of the Cantor set, Math. Systems Theory 6 (1972), 129-132.

4. T. Kimura, Homeomorphisms on zero dimentional spaces, preprint.

5. P. Walters, On the pseudo orbit tracing property and its relationship to stability, The Structure of Attractors in Dynamical Systems, Lecture Notes in Math., vol. 668, Springer-Verlag, Berlin, Heidelberg and New York, 1978, pp. 231-244.

Department of Mathematics, Faculty of Science, Nagoya University, Chikusa-Ku, NAGOYA, 464, JAPAN 